\title{
Generalized strong vector quasi-equilibrium problems with variable ordering structure
}

\author{
Jia-Yu Mao' ${ }^{1}$ San-hua Wang ${ }^{1,2^{*}}$ and Jin-xia Huang
}

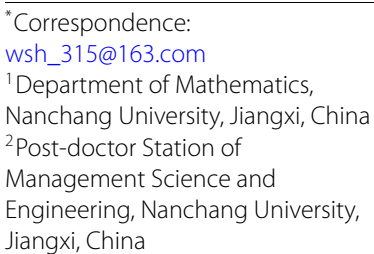

\begin{abstract}
In this paper, two kinds of generalized strong vector quasi-equilibrium problems with variable ordering structure are considered by using the concept of cosmically upper continuity rather than upper semi-continuity for cone-valued mapping. Firstly, a key local property of cosmically upper continuity for cone-valued mapping is discussed. Next, under suitable conditions of cone-continuity and cone-convexity for equilibrium mapping, several existence theorems of solutions and closedness of solution sets are established for these two kinds of generalized strong vector quasi-equilibrium problems with variable ordering structure. Moreover, an example is given to illustrate the validity of our theorems. These results obtained in this paper extend and develop some recent works in this field.
\end{abstract}

MSC: $91 \mathrm{~B} 50 ; 90 \mathrm{C} 33 ; 47 \mathrm{~J} 20$

Keywords: Generalized strong vector quasi-equilibrium problems; Variable ordering structure; Existence of solution; Cosmically upper continuity; Cone-continuity

\section{Introduction}

The so-called equilibrium problem, which is also called generalized Ky Fan minimax inequality, was firstly studied by Blum and Oettli [1] in 1994 in finite-dimensional Euclidean spaces. It provides a brief and unified framework for modeling many problems originating from practice and theory, such as mathematical economics, Nash equilibrium problem, fixed pointed problem, saddle point problem, optimization problem, complementary problem and variational inequality problem, etc. In recent years, equilibrium problems with scalar and vector values objective mappings have been widely studied by researchers, and fruitful results have been established (see, for example, [2-14] and the references therein). Considering in-depth research work, generalized vector quasi-equilibrium problems (GVQEP), in which the constraint set is subject to modification, have been put forward and investigated by many people in different settings. From the existing literature, we can see that there are many meaningful works on the existence of solutions for (GVQEP) (see, e.g., [15-22] and the references therein). As applications of these results, they are applied to study the existence of mathematical programs with equilibrium constraints (MPEC), generalized Nash equilibrium, generalized semi-infinite programs, generalized quasi-variational inequality, and so on. We would like to emphasize here that the importance of research studies on (GVQEP) is justified by the fact that it is a unified model of other several problems. Furthermore, these studies of (GVQEP) can also be applied to

(c) The Author(s) 2019. This article is distributed under the terms of the Creative Commons Attribution 4.0 International License (http://creativecommons.org/licenses/by/4.0/), which permits unrestricted use, distribution, and reproduction in any medium, provided you give appropriate credit to the original author(s) and the source, provide a link to the Creative Commons license, and indicate if changes were made. 
some important real-life problems. For further relevant information, the reader is referred to $[23,24]$ and the references therein.

On the other hand, considering the preference of a decision-maker (the partial order in the object space) may vary with time, Yu [25] introduced in 1974 a new type of vector optimization problem with variable ordering structure, where the ordering structure was represented by a cone-valued mapping, i.e., by a set-valued mapping where the images are cones. In 1992, Chen and Huang [26] generalized it to variational inequality. Afterwards, there have been many works devoted to the study of various equilibrium, optimization, and variational inequality problems with variable ordering structure (see, for example, [27-32] and the references therein). It is worth pointing out that, in these literature sources, the condition of upper semi-continuity for cone-valued mapping has been widely used. However, Borde and Crouzeix [33] gave an example to explain that the concept of the upper semi-continuity for set-valued mapping was actually not suitable for the cone-valued mapping. Very recently, Eichfelder [34] deeply considered various properties for the cone-valued mapping. The author also gave an interesting conclusion, which told us distinctly that the concept of upper semi-continuity for a set-valued mapping is too strict for this special set-valued mapping (cone-valued mapping). In order to deal with this problem, Luc and Penot [35] proposed a modified concept of upper semi-continuity called cosmically upper continuity and analyzed its properties. After that, the cosmically upper continuity conditions for cone-valued mapping have been also used by some authors to discussed vector optimization problems with variable ordering structure. However, to the best of our knowledge, there is no paper dealing with the existence of solutions for equilibrium problem with variable ordering structure by using the concept of cosmically upper continuity.

Inspired and motivated by the works mentioned above, in this paper, we mainly discuss the existence of solutions for generalized strong vector quasi-equilibrium problems with variable ordering structure (for short, GSVQEP) by using the concept of cosmically upper continuity rather than upper semi-continuity for cone-valued mapping. Also, we shall discuss the closedness of solution sets for (GSVQEP).

The rest of the paper is organized as follows. In Sect. 2, some notations are fixed, several well-known concepts and mathematical tools are recalled. In Sect. 3, a local property for cone-valued mapping is discussed under the assumption of cosmically upper continuity. Next, under suitable conditions of cone-continuity and cone-convexity for equilibrium mapping, existence results of solutions and closedness of solution sets are obtained for two kinds of (GSVQEP). Moreover, an example is given to illustrate the validity of our theorem. These results extend and develop some recent works in this field.

\section{Preliminaries}

Throughout this paper, unless otherwise specified, we always suppose that $X$ and $Y$ are two Hausdorff topological vector spaces and $Z$ is a real normed vector space. Let $K$ be a nonempty, closed, and convex subset of $X, E$ be a nonempty subset of $Y$. Let $C: K \rightrightarrows Z$ be a set-valued mapping such that, for all $x \in K, C(x) \subseteq Z$ is a closed, convex, and pointed cone. We denote by $\operatorname{conv}(A), \operatorname{cl}(A)$, and cone $(A)$ the convex, closure, and cone hull of the set $A$, respectively. Since $Z$ is a real normed space, we denote by $B_{l}=\{x \in Z:\|x\|<l\}$ and $S_{l}=\{x \in Z:\|x\|=l\}$ the open ball around $0 \in Z$ with radius $l>0$ and its boundary, respectively. 
Let $F: E \times K \times K \rightrightarrows Z, T: K \times K \rightrightarrows E, Q: K \rightrightarrows K$, and $\widetilde{F}: K \times K \rightrightarrows Z$ be four setvalued mappings with nonempty values. We consider the following two kinds of generalized strong vector quasi-equilibrium problems with variable ordering structure (for short, GSVQEP):

(GSVQEP 1) Find $\bar{x} \in K$ such that $\bar{x} \in Q(\bar{x})$, and for all $y \in Q(\bar{x})$ and $t \in T(\bar{x}, \bar{x})$, one has

$$
F(t, \bar{x}, y) \subseteq C(\bar{x})
$$

(GSVQEP 2) Find $\bar{x} \in K$ such that $\bar{x} \in Q(\bar{x})$, and for any $y \in Q(\bar{x})$, there exists some $t \in T(\bar{x}, \bar{x})$ satisfying

$$
F(t, \bar{x}, y) \subseteq C(\bar{x})
$$

If $X=Y, E=K, T(x, y)=\{x\}, Q(x)=K$, and $F(t, x, y)=\widetilde{F}(x, y)$ for all $x, y \in K$ and $t \in$ $E$, then the two kinds of (GSVQEP) collapse to the following strong vector equilibrium problem (for short, SEVP):

(SVEP) Find $\bar{x} \in K$ such that

$$
\widetilde{F}(\bar{x}, y) \subseteq C(\bar{x}) \quad \text { for all } y \in K
$$

In this section, we will give some necessary background on the continuity for set-valued mapping. Following [36], a set-valued mapping $D: K \rightrightarrows Y$ is said to be upper semicontinuous (for short, u.s.c.) at $x_{0} \in K$ if, for any open set $V \subseteq Y$ with $D\left(x_{0}\right) \subseteq V$, there exists a neighborhood $U$ of $x_{0}$ such that $D(x) \subseteq V$ for all $x \in U \cap K$. D is said to be lower semi-continuous (for short, l.s.c.) at $x_{0} \in K$ if, for any open set $V \subseteq Y$ with $D\left(x_{0}\right) \cap V \neq \emptyset$, there exists a neighborhood $U$ of $x_{0}$ such that $D(x) \cap V \neq \emptyset$ for all $x \in U \cap K$. $D$ is said to be u.s.c. (resp. l.s.c.) on $K$ if it is u.s.c. (resp. l.s.c.) at every point $x \in K$.

Lemma 2.1 ([36, 37]) Let $D: K \rightrightarrows Y$ be a set-valued mapping with nonempty values.

(i) For any given $x_{0} \in K$, if $D\left(x_{0}\right)$ is compact, then $D$ is u.s.c. at $x_{0} \in K$ if and only if, for any net $\left\{x_{\alpha}\right\} \subseteq K$ with $x_{\alpha} \rightarrow x_{0}$ and for any $y_{\alpha} \in D\left(x_{\alpha}\right)$, there exist $y_{0} \in D\left(x_{0}\right)$ and a subnet $\left\{y_{\beta}\right\}$ of $\left\{y_{\alpha}\right\}$ such that $y_{\beta} \rightarrow y_{0}$.

(ii) $D$ is l.s.c. at $x_{0} \in K$ if and only if, for any net $\left\{x_{\alpha}\right\}$ with $x_{\alpha} \rightarrow x_{0}$ and for any $y_{0} \in D\left(x_{0}\right)$, there exists a net $\left\{y_{\alpha}\right\}$ with $y_{\alpha} \in D\left(x_{\alpha}\right)$ such that $y_{\alpha} \rightarrow y_{0}$.

In order to deal with cone-valued mapping, we need the following modified concept of semi-continuity for cone-valued mapping, which is called cosmically upper continuity and was proposed by Luc and Penot in [35]:

A cone-valued mapping $C$ is said to be cosmically upper continuous (for short, c.u.c.) at $x_{0} \in K$ if the mapping $x \rightarrow C(x) \cap \operatorname{cl}\left(B_{1}\right)$ is u.s.c. at $x_{0} \in K$. By analogy to the definition of u.s.c., we say that $C$ is c.u.c. on $K$ if it is c.u.c. at every point $x \in K$.

Remark 2.1 If $C$ is c.u.c. at $x_{0} \in K$, then $-C$ is also c.u.c. at $x_{0} \in K$, and vice versa.

Example 2.1 Considering the following cone-valued mapping $C: \mathbb{R} \rightrightarrows \mathbb{R}^{2}$,

$$
C(x)=\text { cone } \operatorname{conv}\{(1,0),(1, x)\}, \quad \forall x \in \mathbb{R}
$$


Clearly, $C(0)=\left[0,+\infty\left[\times\{0\}\right.\right.$ and $C(x) \subseteq \mathbb{R}^{2}$ is a closed, convex, and pointed cone for each $x \in \mathbb{R}$. Moreover, for each $\delta>0$, we have

$$
C(x) \cap \operatorname{cl}\left(B_{1}\right) \subseteq C(0) \cap \operatorname{cl}\left(B_{1}\right)+B_{\delta}, \quad \forall|x|<\delta .
$$

Noting that $C(x) \cap \operatorname{cl}\left(B_{1}\right)$ is compact, we can conclude easily from the above formula that the mapping $x \rightarrow C(x) \cap \mathrm{cl}\left(B_{1}\right)$ is u.s.c. at $x=0$, i.e., $C$ is c.u.c. at $x=0$.

On the other hand, for any given $\delta>0$ and $x \neq 0$, we can take some $t>0$ such that $t|x|>\delta$. Then, by the definition of $C$, we know $(t, t x) \in C(x)$ but $(t, t x) \notin C(0)+B_{\delta}$. It follows that $C(x) \nsubseteq C(0)+B_{\delta}$. Hence $C$ is not u.s.c. at $x=0$.

In the sequel, we also need the following notions related to cone-continuity and coneconvexity of set-valued mappings. Following [38], a set-valued mapping $D: K \rightrightarrows Z$ is said to be upper $C\left(x_{0}\right)$-continuous (resp. lower $C\left(x_{0}\right)$-continuous) at $x_{0} \in K$ if, for any neighborhood $V$ of $0 \in Z$, there exists a neighborhood $U$ of $x_{0}$ such that $D(x) \subseteq D\left(x_{0}\right)+V+C\left(x_{0}\right)$ (resp. $\left.D\left(x_{0}\right) \subseteq D(x)+V-C\left(x_{0}\right)\right)$ for all $x \in U \cap K . D$ is said to be upper $C(x)$-continuous (resp. lower $C(x)$-continuous) on $K$ if it is upper $C(x)$-continuous (resp. lower $C(x)$ continuous) at every point $x \in K$.

Let $P \subseteq Z$ be a closed, convex, and pointed cone. A set-valued mapping $D: K \rightrightarrows Z$ is said to be upper properly $P$-quasiconvex on $K$ if, for any $u_{1}, u_{2} \in K$ and $t \in[0,1]$, one has

$$
\begin{aligned}
\text { either } & D\left(u_{1}\right) \subseteq D\left(t u_{1}+(1-t) u_{2}\right)+P \\
\text { or } & D\left(u_{2}\right) \subseteq D\left(t u_{1}+(1-t) u_{2}\right)+P .
\end{aligned}
$$

It is said to be lower properly $P$-quasiconvex on $K$ if, for any $u_{1}, u_{2} \in K$ and $t \in[0,1]$, one has

$$
\begin{aligned}
\text { either } & D\left(t u_{1}+(1-t) u_{2}\right) \subseteq D\left(u_{1}\right)-P \\
\text { or } & D\left(t u_{1}+(1-t) u_{2}\right) \subseteq D\left(u_{2}\right)-P .
\end{aligned}
$$

Definition 2.1 ([39]) Let $P \subseteq Z$ be a closed, convex, and pointed cone. A nonempty convex subset $B \subseteq P$ is said to be a base of $P$ if $0 \notin \operatorname{cl}(B)$ and $P=\operatorname{cone}(B)$.

Lemma 2.2 ([39]) For any given closed, convex, and pointed cone $P \subseteq Z$, if $P$ has a bounded closed base, then $P^{\Delta}:=\left\{f \in Z^{*}: \exists a>0, f(x) \geq a\|x\|, \forall x \in P\right\} \neq \emptyset$.

Lemma 2.3 ([40]) Let $H: K \rightrightarrows K$ be a set-valued mapping. Suppose that

(i) for any $x \in K, x \notin \operatorname{conv}(H(x))$;

(ii) for each $y \in K, H^{-1}(y)=\{x \in K: y \in H(x)\}$ is open in $K$;

(iii) there exist a nonempty and compact subset $M$ of $K$ and a nonempty, compact, and convex subset $N$ of $K$ such that, for each $x \in K \backslash M, N \cap H(x) \neq \emptyset$.

Then there exists $x_{0} \in K$ such that $H\left(x_{0}\right)=\emptyset$.

\section{Main results}

In this section, we denote by $\operatorname{Sol}\left(F_{1}\right), \operatorname{Sol}\left(F_{2}\right)$, and $\operatorname{Sol}(\widetilde{F})$ the sets of solutions for (GSVQEP 1), (GSVQEP 2), and (SEVP), respectively.

Firstly, we consider a local property for cone-valued mapping which is c.u.c. 
Lemma 3.1 Let $x_{0} \in K$. Suppose that $C\left(x_{0}\right)$ has a bounded and closed base and $C$ is c.u.c. at $x_{0}$. Then, for any $0<\varepsilon<l$, there exists a neighborhood $U$ of $x_{0}$ such that

$$
\left[C(x)+C\left(x_{0}\right)\right] \cap B_{l} \subseteq C\left(x_{0}\right)+B_{\varepsilon}, \quad \forall x \in U \cap K .
$$

Proof The proof is divided into four steps.

Step 1 . For any $0<\varepsilon<l$ and $\xi \in C\left(x_{0}\right)^{\Delta}$, there exist $r>\varepsilon$ and $c>0$ such that

$$
\xi(v)>c, \quad \forall v \in\left[C\left(x_{0}\right)+B_{\varepsilon}\right] \cap S_{r}
$$

Since $C\left(x_{0}\right)$ has a bounded and closed base, it follows from Lemma 2.2 that $C\left(x_{0}\right)^{\Delta} \neq \emptyset$. Thus, for any $\xi \in C\left(x_{0}\right)^{\Delta} \subseteq Z^{*}$, there exists $a>0$ such that

$$
\xi(v) \geq a\|v\|, \quad \forall v \in C\left(x_{0}\right) .
$$

Let $b=\max \left\{a,\|\xi\|_{*}\right\}+1$, where $\|\xi\|_{*}$ denotes the norm of the linear operator $\xi$. For any $v \in Z$, it follows from $b>\|\xi\|_{*}$ and $\xi \in Z^{*}$ that

$$
|\xi(v)| \leq\|\xi\|_{*} \cdot\|v\|<b\|v\| .
$$

Set $c=b l+1$ and $r=(c+a \varepsilon+b \varepsilon) / a$. We can see that $a(r-\varepsilon)-b \varepsilon=c>0$ and $r>\varepsilon$. For any $v_{0} \in\left[C\left(x_{0}\right)+B_{\varepsilon}\right] \cap S_{r}$, there exist $v_{1} \in C\left(x_{0}\right)$ and $v_{2} \in B_{\varepsilon}$ such that $v_{0}=v_{1}+v_{2}$. It follows from $\left\|v_{0}\right\|=r$ and $v_{2} \in B_{\varepsilon}$ (i.e., $\left\|v_{2}\right\|<\varepsilon$ ) that

$$
\left\|v_{1}\right\|=\left\|v_{0}-v_{2}\right\| \geq\left\|v_{0}\right\|-\left\|v_{2}\right\|>r-\varepsilon>0
$$

Since $v_{1} \in C\left(x_{0}\right)$, applying (2) and (4), we have

$$
\xi\left(v_{1}\right) \geq a\left\|v_{1}\right\|>a(r-\varepsilon)>0 .
$$

We conclude from (3) and $v_{2} \in B_{\varepsilon}$ that $\left|\xi\left(\nu_{2}\right)\right|<b\left\|\nu_{2}\right\|<b \varepsilon$. This together with (5) implies that

$$
\xi\left(v_{0}\right)=\xi\left(v_{1}+v_{2}\right)>a(r-\varepsilon)-b \varepsilon=c .
$$

By the arbitrariness of $v_{0}$, we obtain that (1) is true.

Step 2. cone $\left\{\left[C\left(x_{0}\right)+B_{\varepsilon}\right] \cap S_{r}\right\} \subseteq$ cone $\left\{x \in C\left(x_{0}\right)+B_{\varepsilon}: \xi(x)=c\right\} \subseteq$ cone $\left\{\left[C\left(x_{0}\right)+B_{\varepsilon}\right] \cap S_{l}\right\}$. Let $C_{1}=\operatorname{cone}\left\{\left[C\left(x_{0}\right)+B_{\varepsilon}\right] \cap S_{r}\right\}$ and $C_{2}=\operatorname{cone}\left\{x \in C\left(x_{0}\right)+B_{\varepsilon}: \xi(x)=c\right\}$. It is easy to see that $C_{1}$ and $C_{2}$ are both cones. We claim that $\left\{\left[C\left(x_{0}\right)+B_{\varepsilon}\right] \cap S_{r}\right\} \subseteq C_{2}$. This together with the definition of $C_{1}$ implies that $C_{1} \subseteq C_{2}$. In fact, for any $u_{1} \in\left[C\left(x_{0}\right)+B_{\varepsilon}\right] \cap S_{r}$, it follows from (1) and $u_{1} \in S_{r}$ that $\xi\left(u_{1}\right)>c>0$. Then there exists $0<\lambda_{1}=c / \xi\left(u_{1}\right)<1$ such that

$$
\xi\left(\lambda_{1} u_{1}\right)=\lambda_{1} \xi\left(u_{1}\right)=c
$$

On the other hand, we can see that $u_{1} \in C\left(x_{0}\right)+B_{\varepsilon}$ and $0 \in C\left(x_{0}\right)+B_{\varepsilon}$. This together with the convexity of $C\left(x_{0}\right)+B_{\varepsilon}$ and $0<\lambda_{1}<1$ implies that

$$
\lambda_{1} u_{1} \in C\left(x_{0}\right)+B_{\varepsilon} .
$$


Applying (6), (7) and the definition of $C_{2}$, we can see that $\lambda_{1} u_{1} \in C_{2}$. It is obvious that $u_{1} \in C_{2}$. By the arbitrariness of $u_{1}$, we can get that $\left\{\left[C\left(x_{0}\right)+B_{\varepsilon}\right] \cap S_{r}\right\} \subseteq C_{2}$. Hence, $C_{1} \subseteq C_{2}$.

Let $C_{3}=$ cone $\left\{\left[C\left(x_{0}\right)+B_{\varepsilon}\right] \cap S_{l}\right\}$. Obviously, $C_{2}$ and $C_{3}$ are both cones. If we can prove that $\left\{x \in C\left(x_{0}\right)+B_{\varepsilon}: \xi(x)=c\right\} \subseteq C_{3}$, then we can easily get $C_{2} \subseteq C_{3}$ by the definition of $C_{2}$. In fact, for each $u_{2} \in C\left(x_{0}\right)+B_{\varepsilon}$ satisfying $\xi\left(u_{2}\right)=c$, we claim that $\left\|u_{2}\right\| \geq l$. If it is not true, then we have $\xi\left(u_{2}\right)<b\left\|u_{2}\right\|<b l<c$ from (3), which contradicts $\xi\left(u_{2}\right)=c$. Therefore, $\left\|u_{2}\right\| \geq l$ which implies that there exists $0<\lambda_{2} \leq 1$ such that

$$
\left\|\lambda_{2} u_{2}\right\|=l
$$

On the other hand, $u_{2} \in C\left(x_{0}\right)+B_{\varepsilon}$ and $0 \in C\left(x_{0}\right)+B_{\varepsilon}$. This together with the convexity of $C\left(x_{0}\right)+B_{\varepsilon}$ and $0<\lambda_{2}<1$ implies that

$$
\lambda_{2} u_{2} \in C\left(x_{0}\right)+B_{\varepsilon} .
$$

Applying (8), (9), and the definition of $C_{3}$, it is easy to obtain that $\lambda_{2} u_{2} \in C_{3}$. Obviously, $u_{2} \in C_{3}$. By the arbitrariness of $u_{2}$, we have $\left\{x \in C\left(x_{0}\right)+B_{\varepsilon}: \xi(x)=c\right\} \subseteq C_{3}$, which implies that $C_{2} \subseteq C_{3}$.

Step 3. There exists a neighborhood $U$ of $x_{0}$ such that $C(x) \cap S_{r} \subseteq\left[C\left(x_{0}\right)+B_{\varepsilon}\right] \cap S_{r}$ for all $x \in U \cap K$.

Since $C$ is $c . u . c$. at $x_{0}$, for the preceding positive numbers $\varepsilon, l, c$, and $r$, there exists a neighborhood $U$ of $x_{0}$ such that, for any $x \in U \cap K$,

$$
C(x) \cap \operatorname{cl}\left(B_{r}\right) \subseteq C\left(x_{0}\right) \cap \operatorname{cl}\left(B_{r}\right)+B_{\varepsilon} \subseteq C\left(x_{0}\right)+B_{\varepsilon} .
$$

By taking intersection with the sphere surface $S_{r}$ in both sides of the above formula, we can get

$$
C(x) \cap S_{r} \subseteq\left[C\left(x_{0}\right)+B_{\varepsilon}\right] \cap S_{r}, \quad \forall x \in U \cap K .
$$

Step 4. For any $x \in U \cap K$, we have $\left[C(x)+C\left(x_{0}\right)\right] \cap B_{l} \subseteq C\left(x_{0}\right)+B_{\varepsilon}$.

Applying (10), we can see that

$$
C(x)=\operatorname{cone}\left\{C(x) \cap S_{r}\right\} \subseteq \operatorname{cone}\left\{\left[C\left(x_{0}\right)+B_{\varepsilon}\right] \cap S_{r}\right\}=C_{1} .
$$

This together with $C_{1} \subseteq C_{2}$ implies that $C(x) \subseteq C_{2}$. Furthermore, if we can prove that $C_{2}$ is convex, then we have

$$
C(x)+C\left(x_{0}\right) \subseteq C_{2}+C_{2}=C_{2}, \quad \forall x \in U \cap X .
$$

This together with $C_{2} \subseteq C_{3}$ implies that

$$
C(x)+C\left(x_{0}\right) \subseteq C_{3}, \quad \forall x \in U \cap X .
$$

On the other hand, for any $u \in C_{3} \cap B_{l}$ with $u \neq 0$, we have $0<\|u\|<l$. By the definition of $C_{3}$, there exists $\lambda>0$ such that $\lambda u \in\left[C\left(x_{0}\right)+B_{\varepsilon}\right] \cap S_{l}$. Thus we can see that $\lambda>1$. This 
together with $\lambda u \in C\left(x_{0}\right)+B_{\varepsilon}$ implies that $u \in C\left(x_{0}\right)+B_{\varepsilon}$. Then, by the arbitrariness of $u$, we get

$$
C_{3} \cap B_{l} \subseteq C\left(x_{0}\right)+B_{\varepsilon} .
$$

We conclude from (11) and (12) that, for any $x \in U \cap K$,

$$
\left[C(x)+C\left(x_{0}\right)\right] \cap B_{l} \subseteq C_{3} \cap B_{l} \subseteq C\left(x_{0}\right)+B_{\varepsilon} .
$$

Finally, we show that $C_{2}$ is convex. Since $C_{2}$ is a cone, we only need to prove that, for any $w_{1}, w_{2} \in C_{2} \backslash\{0\}$, one has $w_{1}+w_{2} \in C_{2}$. According to the definition of $C_{2}$, there exist $\lambda_{1}, \lambda_{2}>0$ such that $\lambda_{1} w_{1}, \lambda_{2} w_{2} \in C\left(x_{0}\right)+B_{\varepsilon}$ and $\xi\left(\lambda_{1} w_{1}\right)=\xi\left(\lambda_{2} w_{2}\right)=c$. Let $\lambda=\lambda_{1} /\left(\lambda_{1}+\lambda_{2}\right)$, which implies that $0<\lambda<1$. By the linearity of $\xi$, we have

$$
\xi\left((1-\lambda) \lambda_{1} w_{1}+\lambda \lambda_{2} w_{2}\right)=(1-\lambda) \xi\left(\lambda_{1} w_{1}\right)+\lambda \xi\left(\lambda_{2} w_{2}\right)=(1-\lambda) c+\lambda c=c .
$$

Noting that $C\left(x_{0}\right)+B_{\varepsilon}$ is convex, we can see that $(1-\lambda) \lambda_{1} w_{1}+\lambda \lambda_{2} w_{2} \in C\left(x_{0}\right)+B_{\varepsilon}$. This together with (13) implies that $(1-\lambda) \lambda_{1} w_{1}+\lambda \lambda_{2} w_{2} \in C_{2}$. Hence,

$$
(1-\lambda) \lambda_{1} w_{1}+\lambda \lambda_{2} w_{2}=\frac{\lambda_{1} \lambda_{2}}{\lambda_{1}+\lambda_{2}} w_{1}+\frac{\lambda_{1} \lambda_{2}}{\lambda_{1}+\lambda_{2}} w_{2} \in C_{2},
$$

which implies that $w_{1}+w_{2} \in C_{2}$.

For the simplification of the proof for our main results in this paper, we also need the following lemma.

Lemma 3.2 Let $\left(t_{0}, x_{0}, y_{0}\right) \in E \times K \times K$. Assume that $F$ is lower $\left\{-C\left(x_{0}\right)\right\}$-continuous at $x_{0}$, $C$ is c.u.c. at $x_{0}$, and $C\left(x_{0}\right)$ has a bounded and closed base. Then, for any net $\left\{\left(t_{\alpha}, x_{\alpha}, y_{\alpha}\right)\right\} \rightarrow$ $\left(t_{0}, x_{0}, y_{0}\right)$ with $F\left(t_{\alpha}, x_{\alpha}, y_{\alpha}\right) \subseteq C\left(x_{\alpha}\right)$, we have $F\left(t_{0}, x_{0}, y_{0}\right) \subseteq C\left(x_{0}\right)$.

Proof Suppose to the contrary that there exists $w_{0} \in F\left(t_{0}, x_{0}, y_{0}\right)$ such that $w_{0} \notin C\left(x_{0}\right)$. Since $C\left(x_{0}\right)$ is closed, there exists $\varepsilon>0$ such that

$$
\left[w_{0}+B_{\varepsilon}\right] \cap\left[C\left(x_{0}\right)+B_{\varepsilon}\right]=\emptyset
$$

Applying the lower $\left\{-C\left(x_{0}\right)\right\}$-continuity of $F$, there exists $\alpha_{1}$ such that, for all $\alpha>\alpha_{1}$,

$$
\begin{aligned}
w_{0} \in F\left(t_{0}, x_{0}, y_{0}\right) & \subseteq F\left(t_{\alpha}, x_{\alpha}, y_{\alpha}\right)+B_{\varepsilon}+C\left(x_{0}\right) \\
& \subseteq C\left(x_{\alpha}\right)+B_{\varepsilon}+C\left(x_{0}\right) .
\end{aligned}
$$

This implies that

$$
\left[w_{0}+B_{\varepsilon}\right] \cap\left[C\left(x_{\alpha}\right)+C\left(x_{0}\right)\right] \neq \emptyset, \quad \forall \alpha>\alpha_{1} .
$$

Take any real number $l>\varepsilon$ such that $w_{0}+B_{\varepsilon} \subseteq B_{l}$. Since $C$ is c.u.c. at $x_{0} \in K$ and $C\left(x_{0}\right)$ has a bounded closed base, it follows from Lemma 3.1 that there exists $\alpha_{2}$ such that, for any 
$\alpha>\alpha_{2}$,

$$
\left[C\left(x_{\alpha}\right)+C\left(x_{0}\right)\right] \cap B_{l} \subseteq C\left(x_{0}\right)+B_{\varepsilon} .
$$

Let $\alpha_{0}>\alpha_{1}$ and $\alpha_{0}>\alpha_{2}$. Then, for any $\alpha>\alpha_{0}$, we conclude from (15) and $w_{0}+B_{\varepsilon} \subseteq B_{l}$ that

$$
\left[w_{0}+B_{\varepsilon}\right] \cap\left[C\left(x_{\alpha}\right)+C\left(x_{0}\right)\right] \cap B_{l}=\left[w_{0}+B_{\varepsilon}\right] \cap\left[C\left(x_{\alpha}\right)+C\left(x_{0}\right)\right] \neq \emptyset .
$$

Combining (16) with (17), we can see that $\left[w_{0}+B_{\varepsilon}\right] \cap\left[C\left(x_{0}\right)+B_{\varepsilon}\right] \neq \emptyset$, which contradicts (14). Hence, $F\left(t_{0}, x_{0}, y_{0}\right) \subseteq C\left(x_{0}\right)$.

Remark 3.1 In Lemma 3.2, we use the assumptions that $C$ is c.u.c. and $F$ is lower $\{-C(x)\}$ continuous, which is very different from the ones that $C$ is closed and $F$ is l.s.c. frequently used in the previous literature.

Now, we are ready to present our main results.

Theorem 3.1 Let $F(t, x, x) \subseteq C(x)$ for any $(t, x) \in E \times K$. Assume that $\Omega=\{x \in K: x \in$ $Q(x)$ \} is closed, $Q^{-1}(y)$ is open in $K$ for any $y \in K$, and the following conditions are satisfied:

(i) $C$ is c.u.c. on $K$, and for any $x \in K, C(x)$ has a bounded and closed base;

(ii) $T$ is l.s.c. on $K \times K$ with nonempty and convex values;

(iii) for any $y \in K$, the mapping $(t, x) \rightarrow F(t, x, y)$ is lower $\{-C(x)\}$-continuous on $E \times K$;

(iv) for any $x \in K$, the mapping $(t, y) \rightarrow F(t, x, y)$ is upper properly $C(x)$-quasiconvex on $E \times K$

(v) there exist a nonempty and compact subset $M$ of $K$ and a nonempty, compact, and convex subset $N$ of $K$ such that, for each $x \in K \backslash M$, there exists some $y \in N \cap Q(x)$ such that $F(t, x, y) \nsubseteq C(x)$ for some $t \in T(x, x)$.

Then $\operatorname{Sol}\left(F_{1}\right)$ is nonempty.

Proof Define two set-valued mappings $A, H: K \rightrightarrows K$ as follows: for each $x \in K$,

$$
A(x)=\{y \in K: \exists t \in T(x, x), F(t, x, y) \nsubseteq \subset C(x)\}
$$

and

$$
H(x)= \begin{cases}Q(x) \cap A(x), & x \in \Omega, \\ Q(x), & x \in K \backslash \Omega .\end{cases}
$$

We assert that there exists $\bar{x} \in K$ such that $H(\bar{x})=\emptyset$. If $\bar{x} \in K \backslash \Omega$, then, by the definition of $H$, we have $H(\bar{x})=Q(\bar{x})=\emptyset$, which contradicts the fact that $Q(x) \neq \emptyset$ for all $x \in K$. Hence $\bar{x} \in \Omega$ and $Q(\bar{x}) \cap A(\bar{x})=\emptyset$. This implies that $\bar{x} \in Q(\bar{x})$, and for any $y \in Q(\bar{x})$ and any $t \in$ $T(\bar{x}, \bar{x})$, we have $F(t, \bar{x}, y) \subseteq C(\bar{x})$, and so $\bar{x}$ is a solution of (GSVQEP 1 ). Therefore, it only suffices to prove that there exists $\bar{x} \in K$ such that $H(\bar{x})=\emptyset$.

The proof is divided into three steps.

Step 1. For any $x \in K, x \notin \operatorname{conv}(H(x))$. 
In fact, we can firstly show that $A(x)$ is convex. Indeed, for any $y_{1}, y_{2} \in A(x)$, there exist $t_{1}, t_{2} \in T(x, x)$ such that

$$
F\left(t_{i}, x, y_{i}\right) \nsubseteq \subset C(x), \quad i=1,2 .
$$

Set $y_{\lambda}=\lambda y_{1}+(1-\lambda) y_{2}$ and $t_{\lambda}=\lambda t_{1}+(1-\lambda) t_{2}$ for any $0<\lambda<1$. Since $K$ and $T(x, x)$ are both convex, we have $y_{\lambda} \in K$ and $t_{\lambda} \in T(x, x)$. If $F\left(t_{\lambda}, x, y_{\lambda}\right) \subseteq C(x)$, then, by the upper properly $C(x)$-quasiconvexity of $F(\cdot, x, \cdot)$, we know that there exists $i_{0} \in\{1,2\}$ such that

$$
F\left(t_{i_{0}}, x, y_{i_{0}}\right) \subseteq F\left(t_{\lambda}, x, y_{\lambda}\right)+C(x) \subseteq C(x)+C(x)=C(x) .
$$

This contradicts (18). Hence $F\left(t_{\lambda}, x, y_{\lambda}\right) \nsubseteq \subseteq C(x)$. It follows that $y_{\lambda} \in A(x)$. Then $A(x)$ is convex.

Next, we show that $x \notin \operatorname{conv}(H(x))$. In fact, for any given $x \in K$, since $Q(x)$ and $A(x)$ are convex, we can easily see that $H(x)$ is also convex. Thus, we consider the next two cases. (1) If $x \in \Omega$, then the assumption $F(t, x, x) \subseteq C(x)$ implies $x \notin A(x)$. Hence $x \notin \operatorname{conv}(A(x))$. This together with $H(x) \subseteq A(x)$ implies that $x \notin \operatorname{conv}(H(x))$. (2) If $x \in K \backslash \Omega$, then $x \notin Q(x)$. This together with $\operatorname{conv}(Q(x))=\operatorname{conv}(H(x))$ implies that $x \notin \operatorname{conv}(H(x))$.

Step 2. For all $y \in X, H^{-1}(y)$ is open in $K$.

Indeed, for each $y \in K$, by the definitions of $A$ and $H$, we have

$$
A^{-1}(y)=\{x \in K: \exists t \in T(x, x), F(t, x, y) \nsubseteq C(x)\}
$$

and

$$
\begin{aligned}
H^{-1}(y) & =\left[\Omega \cap Q^{-1}(y) \cap A^{-1}(y)\right] \cup\left[(K \backslash \Omega) \cap Q^{-1}(y)\right] \\
& =Q^{-1}(y) \cap\left[(K \backslash \Omega) \cup A^{-1}(y)\right] .
\end{aligned}
$$

Noting that $\Omega$ is closed and $Q^{-1}(y)$ is open in $K$, it suffices to show that $A^{-1}(y)$ is also open in $K$. Thus, we only need to show that $K \backslash A^{-1}(y)$ is closed. In fact, for any net $\left\{x_{\alpha}\right\} \subseteq$ $K \backslash A^{-1}(y)$ with $x_{\alpha} \rightarrow x_{0} \in K$, if $x_{0} \in A^{-1}(y)$, then there exists $t_{0} \in T\left(x_{0}, x_{0}\right)$ such that

$$
F\left(t_{0}, x_{0}, y\right) \nsubseteq C\left(x_{0}\right)
$$

We conclude from condition (ii) and Lemma 2.1(ii) that there exists a net $\left\{t_{\alpha}\right\}$ such that $t_{\alpha} \rightarrow t_{0}$ with $t_{\alpha} \in T\left(x_{\alpha}, x_{\alpha}\right)$. Since $x_{\alpha} \notin A^{-1}(y)$, we can see that

$$
F\left(t, x_{\alpha}, y\right) \subseteq C\left(x_{\alpha}\right), \quad \forall t \in T\left(x_{\alpha}, x_{\alpha}\right) .
$$

This together with $t_{\alpha} \in T\left(x_{\alpha}, x_{\alpha}\right)$ implies that $F\left(t_{\alpha}, x_{\alpha}, y\right) \subseteq C\left(x_{\alpha}\right)$. Then, by conditions (i), (iii) and Lemma 3.2, we have $F\left(t_{0}, x_{0}, y\right) \subseteq C\left(x_{0}\right)$, which contradicts (19). Hence $x_{0} \notin K \backslash$ $A^{-1}(y)$.

Step 3. There exist a nonempty and compact subset $M$ of $K$ and a nonempty, compact, and convex subset $N$ of $K$ such that, for each $x \in K \backslash M, N \cap H(x) \neq \emptyset$.

Indeed, by condition (v), there exist a nonempty and compact subset $M$ of $K$ and a nonempty, compact, and convex subset $N$ of $K$ such that, for each $x \in K \backslash M$, there exists 
$y \in N \cap Q(x)$ such that $F(t, x, y) \nsubseteq \subseteq C(x)$ for some $t \in T(x, x)$. Hence $y \in Q(x) \cap A(x) \cap N$. It follows that $y \in N \cap H(x)$.

Thus all the conditions of Lemma 2.3 are satisfied. We conclude from Lemma 2.3 that there exists $\bar{x} \in X$ such that $H(\bar{x})=\emptyset$.

In the following, the closedness of the solution set $\operatorname{Sol}\left(F_{1}\right)$ is discussed.

Lemma 3.3 Suppose that the conditions in Theorem 3.1 are all satisfied. Assume that $Q$ is l.s.c. on $K$ and $F$ is lower $\{-C(x)\}$-continuous on $E \times K \times K$. Then $\operatorname{Sol}\left(F_{1}\right)$ is closed.

Proof In fact, for any net $\left\{x_{\alpha}\right\} \subseteq \operatorname{Sol}\left(F_{1}\right)$ with $x_{\alpha} \rightarrow x_{0} \in K$, we can see that $x_{\alpha} \in Q\left(x_{\alpha}\right)$, which implies that $x_{\alpha} \in \Omega$. Since $\Omega$ is closed in $K$, we have $x_{0} \in \Omega$, i.e., $x_{0} \in Q\left(x_{0}\right)$. If $x_{0} \notin \operatorname{Sol}\left(F_{1}\right)$, then there exist some $y_{0} \in Q\left(x_{0}\right)$ and $t_{0} \in T\left(x_{0}, x_{0}\right)$ such that

$$
F\left(t_{0}, x_{0}, y_{0}\right) \nsubseteq \subseteq C\left(x_{0}\right)
$$

Since $Q$ and $T$ are l.s.c. on $K$ and $K \times K$, respectively, we conclude from Lemma 2.1(ii) that there exist $y_{\alpha} \in Q\left(x_{\alpha}\right)$ and $t_{\alpha} \in T\left(x_{\alpha}, x_{\alpha}\right)$ such that $y_{\alpha} \rightarrow y_{0}$ and $t_{\alpha} \rightarrow t_{0}$. Applying the assumption that $x_{\alpha} \in \operatorname{Sol}\left(F_{1}\right)$, we can get $F\left(t, x_{\alpha}, y\right) \subseteq C\left(x_{\alpha}\right)$ for all $y \in Q\left(x_{\alpha}\right)$ and $t \in$ $T\left(x_{\alpha}, x_{\alpha}\right)$. Therefore,

$$
F\left(t_{\alpha}, x_{\alpha}, y_{\alpha}\right) \subseteq C\left(x_{\alpha}\right)
$$

By conditions (i) and (iii), we conclude from Lemma 3.2 and (21) that

$$
F\left(t_{0}, x_{0}, y_{0}\right) \subseteq C\left(x_{0}\right)
$$

which contradicts (20). Hence $x_{0} \in \operatorname{Sol}\left(F_{1}\right)$, which implies that $\operatorname{Sol}\left(F_{1}\right)$ is a closed subset of $K$.

Remark 3.2 Theorem 3.1 is very different from Theorem 4.2 of Lin and Huang [18]. More specifically, (a) in order to establish the existence result of solutions for (GSVQEP 1), Lin and Huang used the upper semi-continuity condition for the cone-valued mapping, while we used here the cosmically upper continuity condition for it; (b) in Theorem 4.2 of Lin and Huang[18], the set-valued mapping $F(\cdot, \cdot, y)$ is assumed to be l.s.c. on $E \times K$, while in Theorem 3.1 of this paper, it is assumed to be lower $\{-C(x)\}$-continuous on $E \times K$.

Remark 3.3 Chen, Yang, and Yu [26] discussed another form of equilibrium problem with variable ordering structure. By applying the scalarization method and the conditions that the cone-valued mappings $C(\cdot)$ and $W(\cdot):=Z \backslash-\operatorname{int} C(\cdot)$ are both u.s.c., they proved an existence theorem of solutions for a single-valued weak vector quasi-equilibrium problem with variable ordering structure. Different from Theorem 3.2 of Chen, Yang, and Yu [26], in Theorem 3.1 of this paper, by using the condition that $C$ is c.u.c., we also obtain the existence result of solutions for a set-valued strong vector quasi-equilibrium problem with variable ordering structure. 
Example 3.1 Let $Z=\mathbb{R}^{2}, X=Y=\mathbb{R}, K=[0, \pi / 2]$, and $E=[0,1]$. For each $x, y \in K$ and $t \in E$, let

$$
\begin{aligned}
& C(x)=\text { cone } \operatorname{conv}\{(1,0),(\cos x, \sin x)\}, \\
& T(x)=[0,1], \quad Q(x)=[0,1], \\
& h(x, y)=((x-y) \cos x,(x-y) \sin x)
\end{aligned}
$$

and

$$
F(t, x, y)= \begin{cases}\operatorname{cone}\{(\cos t x, \sin t x)\}+h(x, y), & x \leq y \\ \operatorname{cone}\{(\cos t x, \sin t x)\} \cap \operatorname{cl}\left(B_{5}\right), & x>y\end{cases}
$$

For any given $y_{0} \in(0, \pi / 2)$ and $t_{0} \in E$, if $x_{0}=y_{0}$, then $h\left(x_{0}, y_{0}\right)=0$, and so

$$
F\left(t_{0}, x_{0}, y_{0}\right)=\operatorname{cone}\left\{\left(\cos t_{0} x_{0}, \sin t_{0} x_{0}\right)\right\}
$$

We can verify that $F\left(\cdot, \cdot, y_{0}\right)$ is not l.s.c. at the point $\left(t_{0}, x_{0}\right)$. In fact, for any neighborhood $V \times U$ of $\left(t_{0}, x_{0}\right)$, there exists $\left(t_{0}, \tilde{x}\right) \in V \times U$ such that $\tilde{x}>x_{0}=y_{0}$. It follows from the definition of $F$ that

$$
F\left(t_{0}, \tilde{x}, y_{0}\right)=\operatorname{cone}\left\{\left(\cos t_{0} \tilde{x}, \sin t_{0} \tilde{x}\right)\right\} \cap \operatorname{cl}\left(B_{5}\right) \subseteq \operatorname{cl}\left(B_{5}\right) .
$$

Clearly, there exist a point $v_{0} \in F\left(t_{0}, x_{0}, y_{0}\right)$ with $\left\|v_{0}\right\|>5$ and a neighborhood $W$ of $v_{0}$ such that $W \cap \operatorname{cl}\left(B_{5}\right)=\emptyset$. This together with (22) implies that $W \cap F\left(t_{0}, \tilde{x}, y_{0}\right)=\emptyset$. It is easy to know that $F\left(\cdot, \cdot, y_{0}\right)$ is not l.s.c. at the point $\left(t_{0}, x_{0}\right)$. Therefore, the condition that $F$ is $l$.s.c. on $E \times K \times K$ is not satisfied in Theorem 4.2 of [18]. So, we are unable to use Theorem 4.2 of [18] to decide the existence of the solutions for (GSVQEP 1).

However, for this example, we can verify that all the conditions in Theorem 3.1 of this paper are satisfied.

Indeed, for each $x \in K$, it is easy to see that $C(x) \subseteq \mathbb{R}^{2}$ is a closed, convex, and pointed cone and $\Omega$ is closed as $\Omega=[0,1]$. For each $y \in K, Q^{-1}(y)$ is open in $K$ because $Q^{-1}(y)=K$ or $Q^{-1}(y)=\emptyset$. In addition, for any $(t, x) \in E \times K=[0,1] \times[0, \pi / 2]$, by the definitions of $C$ and $F$, we have

$$
F(t, x, x) \subseteq C(x)
$$

In the following, we verify that conditions (i)-(v) of Theorem 3.1 are all satisfied. By the assumptions of $C$ and $T$, it is easy to get that conditions (i) and (ii) hold. Thus we consider whether the mapping $(t, y) \rightarrow F(t, x, y)$ is upper properly $C(x)$-quasiconvex on $E \times K$ for any $x \in K$. In fact, let $x \in K, \lambda \in(0,1),\left(t_{1}, y_{1}\right),\left(t_{2}, y_{2}\right) \in E \times K$, and $y_{2} \geq y_{1}$. Set $y_{\lambda}=\lambda y_{1}+(1-\lambda) y_{2}$ and $t_{\lambda}=\lambda t_{1}+(1-\lambda) t_{2}$. It suffices to show that there exists $i_{0} \in\{1,2\}$ such that $F\left(t_{i_{0}}, x, y_{i_{0}}\right) \subseteq F\left(t_{\lambda}, x, y_{\lambda}\right)+C(x)$. By the definition of $C$, we have

$$
(0,0) \in \operatorname{cone}\{(\cos t x, \sin t x)\} \subseteq \operatorname{cone} \operatorname{conv}\{(1,0),(\cos x, \sin x)\}=C(x) .
$$


By the definition of $F$, we can see that $h(x, y) \in-C(x)$. This together with (24) implies that

$$
C(x) \subseteq F(t, x, y)+C(x)
$$

If $x>y_{1}$, from (24) and (25), we can see that

$$
F\left(t_{1}, x, y_{1}\right) \subseteq C(x) \subseteq F\left(t_{\lambda}, x, y_{\lambda}\right)+C(x)
$$

If $x \leq y_{1}$, then $y_{2} \geq y_{\lambda} \geq y_{1}$, and so $h\left(x, y_{\lambda}\right)-h\left(x, y_{1}\right)=\left(\left(y_{1}-y_{\lambda}\right) \cos x,\left(y_{1}-y_{\lambda}\right) \sin x\right) \in-C(x)$. It follows that $h\left(x, y_{1}\right)+C(x) \subseteq h\left(x, y_{\lambda}\right)+C(x)$. This together with (24) implies that

$$
\begin{aligned}
F\left(t_{1}, x, y_{1}\right) & \subseteq h\left(x, y_{1}\right)+C(x) \subseteq h\left(x, y_{\lambda}\right)+C(x) \\
& \subseteq F\left(t_{\lambda}, x, y_{\lambda}\right)+C(x) .
\end{aligned}
$$

Therefore, the mapping $(t, y) \rightarrow F(t, x, y)$ is upper properly $C(x)$-quasiconvex on $E \times K$ for any $x \in K$.

Next, we show that $(t, x) \rightarrow F(t, x, y)$ is lower $\{-C(x)\}$-continuous on $E \times K$ for any given $y \in K$. Let $\left(t_{0}, x_{0}\right) \in E \times K$ and $\varepsilon>0$. We consider the following two cases. a) Assume that $x_{0}>y$. Since $F(t, x, y)=\operatorname{cone}\{(\cos t x, \sin t x)\} \cap \operatorname{cl}\left(B_{5}\right)$ for any $x>y$, it is obvious that $F(\cdot, \cdot, y)$ is lower $\left\{-C\left(x_{0}\right)\right\}$-continuous at $\left(t_{0}, x_{0}\right) \in E \times K$. b) Assume that $x_{0}>y$. We firstly define a vector-valued mapping $g: K \rightarrow Z$ as follows:

$$
g(x)= \begin{cases}h(x, y), & x \leq y, \\ (0,0), & x>y .\end{cases}
$$

Since $x_{0} \leq y$, we conclude from the continuity of $h(\cdot, y)$ and $h(y, y)=(0,0)$ that $g$ is continuous on $K$. Thus there exists a neighborhood $W$ of $x_{0}$ such that, for all $x \in U\left(x_{0}\right)$, $g\left(x_{0}\right) \in g(x)+B_{\varepsilon}$. Hence,

$$
\begin{aligned}
F\left(t_{0}, x_{0}, y\right) & =h\left(x_{0}, y\right)+\operatorname{cone}\left\{\left(\cos t x_{0}, \sin t x_{0}\right)\right\}=g\left(x_{0}\right)+\operatorname{cone}\left\{\left(\cos t x_{0}, \sin t x_{0}\right)\right\} \\
& \subseteq g(x)+B_{\varepsilon}+C\left(x_{0}\right) \subseteq F(t, x, y)+B_{\varepsilon}+C\left(x_{0}\right) .
\end{aligned}
$$

This implies that $(t, x) \rightarrow F(t, x, y)$ is lower $\{-C(x)\}$-continuous on $E \times K$.

Since $K$ is a nonempty, compact, and convex subset of $X$, it is easy to see that condition (v) in Theorem 3.1 is satisfied with $M=N=K$.

Therefore, conditions (i) -(v) in Theorem 3.1 of this paper are all satisfied. Thus, by Theorem 3.1, (GSVQEP 1) has at least one solution in $K$.

In fact, we can also verify that $\bar{x}=1$ is the solution of this example. For all $t \in T(\bar{x})=[0,1]$ and for all $y \in Q(\bar{x})=[0,1]$, we conclude from $y \leq 1=\bar{x}$ and $t \in[0,1]$ that

$$
F(t, \bar{x}, y) \subseteq \operatorname{cone}\{(\cos t, \sin t)\} \subseteq \operatorname{cone} \operatorname{conv}\{(1,0),(\cos 1, \sin 1)\}=C(\bar{x}) .
$$

This indicates that $\bar{x}=1$ solves (GSVQEP 1$)$.

Next, the existence of solutions and the closedness of the solution set for (GSVQEP 2) are discussed. 
Theorem 3.2 Let $X, Y, Z, E, K, C, T, Q$, and $F$ be the same as in Theorem 3.1. Suppose that $E$ is compact and $\Omega$ is closed. If the following conditions are satisfied:

(i) $C$ is c.u.c. on $K$, and for all $x \in K, C(x)$ has a bounded and closed base;

(ii) $T$ is u.s.c. on $K \times K$ with nonempty and closed values;

(iii) for each $y \in K$, the mapping $(t, x) \rightarrow F(t, x, y)$ is lower $\{-C(x)\}$-continuous on $E \times K$;

(iv) for any $(t, x) \in E \times K$, the mapping $y \rightarrow F(t, x, y)$ is upper properly $C(x)$-quasiconvex on $K$;

(v) there exist a nonempty and compact subset $M$ of $K$ and a nonempty, compact, and convex subset $N$ of $K$ such that, for each $x \in K \backslash M$, there exists some $y \in N \cap Q(x)$ such that $F(t, x, y) \nsubseteq C(x)$ for all $t \in T(x, x)$.

Then $\operatorname{Sol}\left(F_{2}\right)$ is nonempty.

Proof For each $x \in K$, let

$$
A(x)=\{y \in K: \forall t \in T(x, x), F(t, x, y) \nsubseteq C(x)\}
$$

and

$$
H(x)= \begin{cases}Q(x) \cap A(x), & x \in \Omega, \\ Q(x), & x \in K \backslash \Omega .\end{cases}
$$

Similar to the analysis as Theorem 3.1, it suffices to be proved that there exists $\bar{x} \in K$ such that $H(\bar{x})=\emptyset$.

The proof is divided into three steps.

Step 1. For all $x \in K, x \notin \operatorname{conv}(H(x))$.

In fact, for every $t \in T(x, x)$, by the similar arguments for the convexity of $A$ in the proof of Theorem 3.1, we can show that $A(x)$ is convex for each $x \in K$. In the same way as in Theorem 3.1, we can show that $x \notin \operatorname{conv}(H(x))$.

Step 2. For all $y \in X, H^{-1}(y)$ is open in $K$.

By the definition of $A$ and $H$, we can see that $A^{-1}(y)=\{x \in K: \forall t \in T(x, x), F(t, x, y) \nsubseteq$ $C(x)\}$ and $H^{-1}(y)=Q^{-1}(y) \cap\left[(K \backslash \Omega) \cup A^{-1}(y)\right]$. Similar to the proof of openness of $H^{-1}(y)$ in Theorem 3.1, it suffices to show that $K \backslash A^{-1}(y)$ is closed.

In fact, for any net $\left\{x_{\alpha}\right\} \subseteq K \backslash A^{-1}(y)$ with $x_{\alpha} \rightarrow x_{0} \in K$, we have $x_{\alpha} \notin A^{-1}(y)$. Thus, for each $\alpha$, there exists some $t_{\alpha} \in T\left(x_{\alpha}, x_{\alpha}\right) \subseteq E$ such that

$$
F\left(t_{\alpha}, x_{\alpha}, y\right) \subseteq C\left(x_{\alpha}\right)
$$

Since $E$ is compact, we can see that $T$ is u.s.c. with compact values. By Lemma 2.1(i), there exists a subnet of $\left\{t_{\alpha}\right\}$ which converges to $t_{0} \in T\left(x_{0}, x_{0}\right)$. Without loss of generality, we assume that $t_{\alpha} \rightarrow t_{0}$. It follows from (26) and Lemma 3.2 that $F\left(t_{0}, x_{0}, y\right) \subseteq C\left(x_{0}\right)$. Hence, $x_{0} \in K \backslash A^{-1}(y)$, which implies that $K \backslash A^{-1}(y)$ is closed.

Step 3. There exist a nonempty and compact subset $M$ of $K$ and a nonempty, convex, and compact subset $N$ of $K$ such that, for each $x \in K \backslash M, N \cap H(x) \neq \emptyset$.

Indeed, by condition (v), there exist a nonempty compact subset $M$ of $K$ and a nonempty compact convex subset $N$ of $K$ such that, for each $x \in K \backslash M$, there exists some $y \in N \cap Q(x)$ such that $F(t, x, y) \nsubseteq C(x)$ for all $t \in T(x, x)$. It follows that $y \in Q(x) \cap A(x)$, and so $y \in$ $N \cap H(x)$. 
Applying Lemma 2.3, there exists $\bar{x} \in K$ such that $H(\bar{x})=\emptyset$.

Lemma 3.4 Let $\operatorname{Sol}\left(F_{2}\right) \neq \emptyset$. Suppose that $Q$ is l.s.c. on $K, T$ is u.s.c. on $K \times K$ with compact values, and $F$ is lower $\{-C(x)\}$-continuous on $E \times K \times K$. Then $\operatorname{Sol}\left(F_{2}\right)$ is closed.

Proof In fact, for any net $\left\{x_{\alpha}\right\} \subseteq \operatorname{Sol}\left(F_{2}\right)$ with $x_{\alpha} \rightarrow x_{0} \in K$, we need to prove that $x_{0} \in$ $\operatorname{Sol}\left(F_{2}\right)$. If it is not true, then there exists $y_{0} \in Q\left(x_{0}\right)$ such that, for all $t \in T\left(x_{0}, x_{0}\right)$,

$$
F\left(t, x_{0}, y_{0}\right) \nsubseteq \subset C\left(x_{0}\right)
$$

Since $y_{0} \in Q\left(x_{0}\right)$ and $Q$ is l.s.c. on $K$, applying Lemma 2.1(ii), there exists $y_{\alpha} \in Q\left(x_{\alpha}\right)$ such that $y_{\alpha} \rightarrow y_{0}$. We conclude from $x_{\alpha} \in \operatorname{Sol}\left(F_{2}\right)$ and $y_{\alpha} \in Q\left(x_{\alpha}\right)$ that there exists $t_{\alpha} \in T\left(x_{\alpha}, x_{\alpha}\right)$ such that

$$
F\left(t_{\alpha}, x_{\alpha}, y_{\alpha}\right) \subseteq C\left(x_{\alpha}\right)
$$

Noting that $T$ is u.s.c. with compact values, by Lemma 2.1(i), there exists a subnet of $\left\{t_{\alpha}\right\}$ which converges to $t_{0} \in T\left(x_{0}, x_{0}\right)$. Without loss of generality, let $t_{\alpha} \rightarrow t_{0}$. It follows from (28) and Lemma 3.2 that $F\left(t_{0}, x_{0}, y_{0}\right) \subseteq C\left(x_{0}\right)$, which contradicts (27). Thus $x_{0} \in \operatorname{Sol}\left(F_{2}\right)$. This implies that $\operatorname{Sol}\left(F_{2}\right)$ is closed.

Remark 3.4 If $K$ is a nonempty, compact, and convex subset of $X$, we can take $M=N=K$. Then, condition (v) in Theorems 3.1 and 3.2 is satisfied trivially. Furthermore, if $Q$ is u.s.c. with nonempty, closed, and convex values, then the set $\Omega=\{x \in K: x \in Q(x)\}$ is closed.

Remark 3.5 In the situation that $Q(x) \equiv K$ for all $x \in K$, Fu and Wang [29] obtained an existence theorem of solutions for (GSVQEP 2)-Theorem 3.1, which is very different from Theorem 3.2 of this paper. Above all the differences, the continuity condition on the conevalued mapping $C$ is the biggest one. Indeed, the cone-valued mapping $C$ is assumed to be u.s.c. in Theorem 3.1 of Fu and Wang [29], while it is assumed to be c.u.c. in Theorem 3.2 of this paper.

By applying Theorem 3.1, we can get the following corollary.

Corollary 3.1 Let $X, Z, K$, and $C$ be same as in Theorem 3.1. Let $\widetilde{F}: K \times K \rightrightarrows Z$ be a set-valued mapping. Suppose that the following conditions are satisfied:

(i) $C$ is c.u.c. on $K$, and for all $x \in K, C(x)$ has a bounded and closed base;

(ii) for all $x \in K, \widetilde{F}(x, x) \subseteq C(x)$;

(iii) for each $x \in K$, the mapping $y \rightarrow \widetilde{F}(x, y)$ is upper properly $C(x)$-quasiconvex on $K$;

(iv) for each $y \in K$, the mapping $x \rightarrow \widetilde{F}(x, y)$ is lower $\{-C(x)\}$-continuous on $K$;

(v) there exist a nonempty and compact subset $M$ of $K$ and a nonempty, compact, and convex subset $N$ of $K$ such that, for each $x \in K \backslash M$, there exists $y \in N \cap Q(x)$ satisfying $\widetilde{F}(x, y) \nsubseteq C(x)$.

Then the solution set $\operatorname{Sol}(\widetilde{F})$ is nonempty and closed.

Proof Let $Y=X$ and $E=K$. For each $x, y \in K$, let

$$
Q(x)=K, \quad T(x, y)=\{x\} \quad \text { and } \quad F(t, x, y)=\widetilde{F}(x, y) .
$$


It is easy to verify that conditions (i)-(v) of Theorem 3.1 are all satisfied. Then, by Theorem 3.1, there exists $\bar{x} \in K$ such that

$$
\widetilde{F}(\bar{x}, y) \subseteq C(\bar{x}), \quad \forall y \in Q(\bar{x})=K .
$$

Hence, $\bar{x}$ is a solution of (SVEP).

Next, we show that $\operatorname{Sol}(\widetilde{F})$ is closed. In fact, for any net $\left\{x_{\alpha}\right\} \subseteq \operatorname{Sol}(\widetilde{F})$ with $x_{\alpha} \rightarrow x_{0} \in K$, we can see that

$$
\widetilde{F}\left(x_{\alpha}, y\right) \subseteq C\left(x_{\alpha}\right), \quad \forall y \in K
$$

Then, for each $y \in X$, we conclude from (i), (iv), and Lemma 3.2 that $\widetilde{F}\left(x_{0}, y\right) \subseteq C\left(x_{0}\right)$. This implies that $x_{0} \in \operatorname{Sol}(\widetilde{F})$. Hence, $\operatorname{Sol}(\widetilde{F})$ is closed.

\section{Conclusions}

The aim of this paper is to investigate generalized strong vector quasi-equilibrium problems with variable ordering structure, which has already been studied in the previous literature. However, we would like to point out that the concept of upper semi-continuity, which is widely used for cone-valued mapping in many results, just as Borde and Crouzeix [33] pointed out, is actually not suitable for the cone-valued mapping because of its strange characterization. And so, the value of those previous results will be heavily discounted. In order to deal with cone-valued mapping, some papers consider the existence of solutions for equilibrium problems under the conditions that cone-valued mapping is closed and the equilibrium mapping is continuous vector-valued mapping or lower semi-continuous set-valued mapping. Different from these existing literature sources, we establish the existence of solutions for (GSVQEP) by using the assumption that the equilibrium mapping is cone-continuous. Another difficulty of this article is the continuity condition of cone-valued mapping. Without using the upper semi-continuity, we consider the inclusion relationship of bases for these cones and further obtain a local property by using the modified concept called cosmically upper continuity. Also, this conclusion plays a key role in the existence theorems of solution for these (GSVQEP). Moreover, an example is given to illustrate the validity of our theorems. These results obtained in this paper extend and develop some recent works in this field.

Acknowledgements

Not applicable.

Funding

This work was supported by the National Natural Science Foundation of China (Grant Nos. 11661055, 11201216,

11661056, 11771198, and 11801257), the Natural Science Foundation of Jiangxi Province (Grant No. 20151BAB201020),

the China Postdoctoral Science Foundation (Grant No. 2015M582047), the Postdoctoral Science Foundation of Jiangxi

Province (Grant No. 2016KY58), and the Science Fund of the Education Department of Jiangxi Province (Grant no. GJJ160063)

Availability of data and materials

Not applicable.

Competing interests

The authors declare that they have no competing interests.

Authors' contributions

MJY and WSH carried out the design of the study and performed the analysis. HJX participated in its design and coordination. All authors read and approved the final manuscript. 


\section{Publisher's Note}

Springer Nature remains neutral with regard to jurisdictional claims in published maps and institutional affiliations.

\section{Received: 11 January 2019 Accepted: 8 April 2019 Published online: 29 April 2019}

\section{References}

1. Blum, E., Oettli, W.: From optimization and variational inequalities to equilibrium problems. Math. Stud. 63, 123-145 (1994)

2. Flores-Bazán, F: Existence theorems for generalized noncoercive equilibrium problems: quasiconvex case. SIAM J. Optim. 11, 675-790 (2000)

3. Flores-Bazán, F., Flores-Bazán, F.: Vector equilibrium problems under asymptotic analysis. J. Glob. Optim. 26, 141-166 (2003)

4. Anh, L.Q., Bantaojai, T., Duc, N.P., Duy, T.Q., Wangkeeree, R.: Convergence of solutions to lexicographic equilibrium problems. Appl. Numer. Optim. 1, 39-51 (2019)

5. Ceng, L.C.: Convergence analysis of a Mann-like iterative algorithm in reflexive Banach spaces. Appl. Set-Valued Anal. Optim. 1, 1-18 (2019)

6. Saidi, A., Chadli, O., Yao, J.C.: Second order nonlinear evolution equations with time dependent pseudomonotone and quasimonotone operators: an equilibrium problem approach. Appl. Anal. Optim. 1, 245-259 (2017)

7. Bianchi, M., Pini, R.: Coercivity conditions for equilibrium problems. J. Optim. Theory Appl. 124, 79-92 (2005)

8. Iusem, A.N., Sosa, W.: New existence results for equilibrium problems. Nonlinear Anal. TMA 52, 621-635 (2003)

9. Iusem, A.N., Kassay, G., Sosa, W.: On certain conditions for the existence of solutions of equilibrium problems. Math. Program. 116, 259-273 (2009)

10. Gutiérrez, C., Kassay, G., Novo, V., Ródenas-Pedregosa, J.L.: Ekeland variational principles in vector equilibrium problems. SIAM J. Optim. 27, 2405-2425 (2017)

11. Ansari, Q.H.: Vector equilibrium problems and vector variational inequalities. In: Giannessi, F. (ed.) Vector Variational Inequalities and Vector Equilibria, pp. 1-16. Kluwer Academic, Dordrecht (2000)

12. Ansari, Q.H., Farajzadeh, A.P., Schaible, S.: Existence of solutions of strong vector equilibrium problems. Taiwan. J. Math. 16, 165-178 (2012)

13. Chen, J., Liou, Y.C., Wan, Z., Yao, J.C.: A proximal point method for a class of monotone equilibrium problems with linear constraints. Oper. Res. 15, 275-288 (2015)

14. Kassay, G.: On equilibrium problems. In: Chinchuluun, A., Pardalos, P., Enkhbat, R., Tseveendorj, I. (eds.) Optimization and Optimal Control: Theory and Applications, vol. 39, pp. 55-83. Springer, Berlin (2010)

15. Guu, S.M. Li, J.: Vector quasi-equilibrium problems: separation, saddle points and error bounds for the solution set. J. Glob. Optim. 58, 751-767 (2014)

16. Castellani, M., Giuli, M.: An existence result for quasi-equilibrium problems in separable Banach spaces. J. Math. Anal. Appl. 425, 85-95 (2015)

17. Alleche, B., Rădulescu, V.D.: Solutions and approximate solutions of quasi-equilibrium problems in Banach spaces. J. Optim. Theory Appl. 170, 629-649 (2016)

18. Lin, L.J., Huang, Y.J.: Generalized vector quasi-equilibrium problems with applications to common fixed point theorems and optimization problems. Nonlinear Anal. TMA 66, 1275-1289 (2007)

19. Zhang, W.B., Shan, S.Q., Huang, N.J.: Existence of solutions for generalized vector quasi-equilibrium problems in abstract convex spaces with applications. Fixed Point Theory Appl. 2015, 29 (2015)

20. Ansari, Q.H., Flores-Bazán, F.: Generalized vector quasi-equilibrium problems with applications. J. Math. Anal. Appl. 277, 246-356 (2003)

21. Hai, N.X., Khanh, P.Q.: Existence of solutions to general quasiequilibrium problems and applications. J. Optim. Theory Appl. 133, 317-327 (2007)

22. Ansari, Q.H., Yao, J.C.: Systems of vector quasi-equilibrium problems and their applications. In: Burachik, R.S., Yao, J.C. (eds.) Variational Analysis and Generalized Differentiation in Optimization and Control, vol. 47, pp. 1-42. Springer, New York (2010)

23. Luo, Z.Q., Pang, J.S., Ralph, D.: Mathematical Programs with Equilibrium Constraints. Cambridge University Press, Cambridge (1996)

24. Anh, L.Q., Hung, N.V.: Levitin-Polyak well-posedness for strong bilevel vector equilibrium problems and applications to traffic network problems with equilibrium constraints. Positivity 22, 1223-1239 (2018)

25. Yu, P.L.: Cone convexity, cone extreme points, and nondominated solutions in decision problems with multiobjectives. J. Optim. Theory Appl. 14, 319-377 (1974)

26. Chen, G.Y., Huang, X.X., Yang, X.Q.: Vector Optimization, Set-Valued and Variational Analysis. Springer, Berlin (2005)

27. Li, S.J., Teo, K.L., Yang, X.Q., Wu, S.Y.: Gap functions and existence of solutions to generalized vector quasi-equilibrium problems. J. Glob. Optim. 34, 427-440 (2006)

28. Mastroeni, G.: On the image space analysis for vector quasi-equilibrium problems with a variable ordering relation. J. Glob. Optim. 53, 203-214 (2012)

29. Fu, J.Y., Wang, S.H.: Generalized strong vector quasi-equilibrium problem with domination structure. J. Glob. Optim. 55, 839-847 (2013)

30. Farajzadeh, A., Lee, B.S., Plubteing, S.: On generalized quasi-vector equilibrium problems via scalarization method. J. Optim. Theory Appl. 168, 584-599 (2016)

31. Eichfelder, G.: Optimal elements in vector optimization with a variable ordering structure. J. Optim. Theory Appl. 151, 217-240 (2011)

32. Anh, L.Q., Hien, D.V.: On well-posedness for parametric vector quasiequilibrium problems with moving cones. Appl. Math. 61, 651-668 (2016)

33. Borde, J., Crouzeix, J.P.: Continuity properties of the normal cone to the level sets of a quasiconvex function. J. Optim. Theory Appl. 66, 415-429 (1990)

34. Eichfelder, G.: Variable Ordering Structures in Vector Optimization. Springer, Berlin (2014)

35. Luc, D.T., Penot, J.P.: Convergence of asymptotic directions. Trans. Am. Math. Soc. 353, 4095-4121 (2001) 
36. Aubin, J.P., Ekeland, I.: Applied Nonlinear Analysis. Wiley, New York (1984)

37. Göpfert, A., Riahi, H., Tammer, C., Zălinescu, C.: Variational Methods in Partially Ordered Spaces. Springer, New York (2003)

38. Tan, N.X.: On the existence of solutions of quasivariational inclusion problems. J. Optim. Theory Appl. 123, 619-638 (2004)

39. John, J.: Mathematical Vector Optimization in Partially Ordered Linear Spaces. Peter Lang, Frankfurt am Main (1986)

40. Deguire, P., Tan, K.K., Yuan, X.Z.: The study of maximal elements, fixed points for $\mathrm{L}_{\mathrm{S}}$-majorized mappings and their applications to minimax and variational inequalities in product topological spaces. Nonlinear Anal. TMA 37, 933-951 (1999)

\section{Submit your manuscript to a SpringerOpen ${ }^{\mathcal{O}}$} journal and benefit from:

- Convenient online submission

Rigorous peer review

- Open access: articles freely available online

- High visibility within the field

- Retaining the copyright to your article

Submit your next manuscript at $\gg$ springeropen.com 